Research Article

\title{
Improved Outcomes following the Establishment of a Neurocritical Care Unit in Saudi Arabia
}

\author{
Ibrahim Soliman ${ }^{D},{ }^{1}$ Waleed Tharwat Aletreby ${ }^{D},{ }^{1}$ Fahad Faqihi, ${ }^{1}$ Nasir Nasim Mahmood, ${ }^{1}$ \\ Omar E. Ramadan, ${ }^{1,2}$ Ahmad Fouad Mady, ${ }^{1,3}$ Babar Kahlon, ${ }^{4}$ Abdulrahman Alharthy, \\ Peter Brindley, ${ }^{5}$ and Dimitrios Karakitsos $\mathbb{D D}^{1,6}$
}

\author{
${ }^{1}$ Neurocritical Care Unit, Critical Care Department, King Saud Medical City, Riyadh, Saudi Arabia \\ ${ }^{2}$ Anesthesia and Critical Care Department, Ain Shams University, Cairo, Egypt \\ ${ }^{3}$ Anesthesia and Critical Care Department, Tanta University, Tanta, Egypt \\ ${ }^{4}$ Department of Neurosurgery, King Saud Medical City, Riyadh, Saudi Arabia \\ ${ }^{5}$ Critical Care Department, University of Alberta, Canada \\ ${ }^{6}$ Department of Critical Care, Keck School of Medicine, USC, Los Angeles, CA, USA
}

Correspondence should be addressed to Ibrahim Soliman; ibrahimrefaat@hotmail.com

Received 1 November 2017; Revised 27 April 2018; Accepted 20 June 2018; Published 18 July 2018

Academic Editor: Robert Boots

Copyright (C) 2018 Ibrahim Soliman et al. This is an open access article distributed under the Creative Commons Attribution License, which permits unrestricted use, distribution, and reproduction in any medium, provided the original work is properly cited.

\begin{abstract}
Background. Dedicated neurocritical care units have dramatically improved the management and outcome following brain injury worldwide. Aim. This is the first study in the Middle East to evaluate the clinical impact of a neurocritical care unit (NCCU) launched within the diverse clinical setting of a polyvalent intensive care unit (ICU). Design and Methods. A retrospective before and after cohort study comparing the outcomes of neurologically injured patients. Group one met criteria for NCCU admission but were admitted to the general ICU as the NCCU was not yet operational (group 1). Group two were subsequently admitted thereafter to the NCCU once it had opened (group 2). The primary outcome was all-cause ICU and hospital mortality. Secondary outcomes were ICU length of stay (LOS), predictors of ICU and hospital discharge, ICU discharge Glasgow Coma Scale (GCS), frequency of tracheostomies, ICP monitoring, and operative interventions. Results. Admission to NCCU was a significant predictor of increased hospital discharge with an odds ratio of 2.3 (95\% CI: $1.3-4.1 ; p=0.005)$. Group 2 ( $n=208$ patients) compared to Group 1 ( $n=364$ patients) had a significantly lower ICU LOS (15 versus 21.4 days). Group 2 also had lower ICU and hospital mortality rates (5.3\% versus $10.2 \%$ and $9.1 \%$ versus $19.5 \%$, respectively; all $p<0.05$ ). Group 2 patients had higher discharge GCS and underwent fewer tracheostomies but more interventional procedures (all $p<0.05$ ). Conclusion. Admission to NCCU, within a polyvalent Middle Eastern ICU, was associated with significantly decreased mortality and increased hospital discharge.
\end{abstract}

\section{Introduction}

Neurocritical care (NCC) is an expanding subspecialty within critical care medicine while NCC board certification has been offered since $2007[1,2]$. NCC units (NCCUs) have become more widespread and have typically evolved from larger multidisciplinary intensive care units (ICUs) into freestanding units. The goal of the NCCU is to optimize care for brain- and spine-injured patients, who can be vulnerable to physiological and biochemical perturbations [3,4]. Accordingly, a dedicated
NCCU-which includes specialized team, protocols, monitoring, imaging, and expertise-may result in less secondary injury and better outcomes [5-7].

There is growing evidence regarding the benefits associated with NCCU-based care for brain-injured patients. These include shorter hospital length of stay and/or better neurological and functional outcomes for all comers [8-12]. Better outcomes have also been reported for specific disease states: cerebral hemorrhage (ICH) [13], acute ischemic strokes [14], subarachnoid hemorrhage (SAH), and traumatic spinal 
cord and brain injuries [15]. A dedicated NCCU might also be associated with more appropriate resource utilization [14], better adherence to protocols [16], better chart documentation [11], and readmission rates [17]. If so then objective data are important as it could provide justification and leverage for institutions eager to start their own NCCU [18]. Thus far, the vast majority of the NCCU studies have come from North America and Europe, whereas there are scarce data from other nations. This is the first study in the Middle East that evaluated the impact of a newly launched NCCU on the outcome of neurologically injured patients, within the largest polyvalent ICU department in the Middle East.

\section{Patients and Methods}

This study was part of a NCCU performance audit and was approved by the Total Quality Management (TQM) of King Saud Medical City (KSMC). KSMC is the largest ministry of health tertiary referral hospital in Riyadh, Kingdom of Saudi Arabia. The polyvalent KSMC ICU department is the largest in the Middle East (130 operational beds). It is a closed ICU operated $24 / 7$ by consultant intensivists, with an in-house critical care fellow or resident at all times, baring a patient: physician ratio of $12: 1$ and patient: nurse ratio of $1: 1$. In this retrospective cohort study, we compared two time periods: a period of one year prior to the NCCU launching (January 1st to December 31st, 2016) versus a period of nine months after the NCCU was fully operational (January 1st to September 30th, 2017). Patients from the former time period were designated as Group 1, while patients from the latter period were designated Group 2. The latter group included in all neurologically injured patients admitted to the NCCU. In contrast, Group 1 included all neurologically injured patients admitted to the general ICU (since NCCU was not operating at that time) but who fulfilled NCCU admission criteria. These NCCU admission criteria, also served as the study's inclusion criteria, were as follows:

(1) Need for intracranial pressure (ICP) monitoring

(2) Need for advanced neuromonitoring

(3) Need for frequent clinical monitoring due to concerns of neurologic deterioration (including spinal injury)

(4) Subarachnoid hemorrhage patients in the vasospasm time window (day 1-14 post-SAH)

(5) Complex neurosurgery cases immediately after procedure (as determined by the surgeon)

(6) Acute stroke after thrombolytic therapy as well as neuroradiological and/or surgical interventions.

Exclusion criteria applied to both groups were as follows: age $\leq 18$ years old, patients admitted for brain death declaration or in need of solely palliative care, and patients with Do Not Resuscitate (DNR) order. We also excluded patients isolated for infectious conditions (i.e., bacterial meningitis, viral infections, tuberculosis etc.) from Group 1, as the NCCU has no isolation rooms at present, and Group 2 included no such patients. The primary outcome was all-cause mortality and hospital all-cause mortality. Secondary outcomes were ICU length of stay (LOS), identify predictors of ICU and hospital discharge, ICU discharge Glasgow Coma Scale (GCS), as well as the frequency of tracheostomies, ICP monitoring, and operative neurosurgical interventions such as ventriculostomies, craniotomies for hematoma evacuation or removal of contusion, and last tier decompressive craniectomy in TBI and malignant stroke $[19,20]$. The study conformed to the principles outlined in the Declaration of Helsinki and was approved by the ICU Ethics Committee.

\section{Statistical Analysis}

Demographic and clinical data were collected retrospectively for all patients from the departmental electronic database and included age, gender, acute physiology, and chronic health evaluation (APACHE) four score and admission diagnosis. Also, we retrieved data of operative interventions in the ICU, ICU LOS, discharge GCS and airway status as well as ICU and hospital outcome. All discrete variables were reported as number (\%) and compared with the chi-square test. Continuous variables were reported as mean $\pm \mathrm{SD}$ and compared with the $t$-test, accounting for unequal sample sizes (Welch's $t$-test) [21]. All tests were two-sided and considered to be statistically significant when $p$ value was $<0.05$.

In a logistic regression analysis to identify predictors of ICU and hospital discharge [22], we used ICU or hospital discharge as a binary outcome measure and admission to NCCU as a predictor adjusted for age, gender, ICU LOS, and APACHE 4 score, whether the patient had experienced trauma or not, and whether an operative intervention occurred or not. The prediction models used enter method with enter $p$ value of $<0.1$ and tested for goodness of fit with Hosmer-Lemeshow test, the calibration of each model was evaluated by the area under the curve (AUC) of receiver operator characteristics (ROC) curve, accepted as good if the AUC $\geq 0.7$. All statistical tests were carried out by SPSS ${ }^{\odot}$ version 21 for Windows (SPSS Inc. Chicago, Illinois, USA).

\section{Results}

In 2016, a total of 2442 patients were admitted to the polyvalent ICU. Of those, 364 patients fulfilled inclusion criteria for NCCU admission. Since no NCCU yet existed, they were admitted in the polyvalent ICU and represented Group 1 in our study. In 2017, 1765 patients were admitted to the ICU in 9 months, with 208 patients admitted to the NCCU and therefore designated as Group 2. The comparative demographics of Groups 1 and 2 are presented in Table 1.

ICU mortality in Group 2 (5.3\%) was significantly lower than Group $1(10.2 \%)(p=0.034)$, likewise, hospital mortality was significantly lower in Group 2 compared to Group $1(9.1 \%$ versus $19.5 \%, p=0.001)$, (Table 2$)$. The most common causes of death in Group 1 in the ICU were acute respiratory distress syndrome (ARDS) 43\%, followed by brain herniation $30 \%$, and then sepsis and septic shock $27 \%$, whereas Group 2 ICU mortality was mostly due to ARDS $45 \%$, sepsis and septic shock $36 \%$, and brain herniation $19 \%$. Withdrawal of care, however, was statistically similar in both 
TABle 1: Study demographics.

\begin{tabular}{|c|c|c|c|}
\hline Variable & Group $1(n=364)$ & Group $2(n=208)$ & $p$ value \\
\hline Age (years; mean $\pm \mathrm{SD}$ ) & $39.5 \pm 18.1$ & $40.3 \pm 17.9$ & 0.6 \\
\hline Males $(n(\%))$ & $301(82.7 \%)$ & $165(79.3 \%)$ & 0.3 \\
\hline APACHE $4($ mean \pm SD) & $67.9 \pm 22.2$ & $70.9 \pm 22.5$ & 0.1 \\
\hline \multicolumn{4}{|l|}{ Diagnosis: } \\
\hline Trauma (n (\%)) & $257 / 364(70.6 \%)$ & $145 / 208(69.7 \%)$ & 0.9 \\
\hline (i) Polytrauma & $129 / 257(50.2 \%)$ & 74/145 (51\%) & 0.96 \\
\hline With TBI & $104 / 129(80.6 \%)$ & $59 / 74(79.7 \%)$ & 0.97 \\
\hline With spinal cord injury & 25/129 (19.4\%) & 15/74 (20.3\%) & 0.97 \\
\hline (ii) Isolated head injury & $128 / 257(49.8 \%)$ & $71 / 145(49 \%)$ & 0.96 \\
\hline Brain contusion & $32 / 128(25 \%)$ & $23 / 71(32.4 \%)$ & 0.3 \\
\hline $\mathrm{EDH}$ & $11 / 128(8.6 \%)$ & 7/71 (9.9\%) & 0.96 \\
\hline SDH & 6/128 (4.7\%) & 4/71 (5.6\%) & 0.95 \\
\hline SAH & $15 / 128(11.7 \%)$ & $11 / 71(15.5 \%)$ & 0.6 \\
\hline Diffuse brain injury & $64 / 128(50 \%)$ & $34 / 71(47.9 \%)$ & 0.9 \\
\hline Nontraumatic (n (\%)) & $107 / 364(29.4 \%)$ & $63 / 208(30.3 \%)$ & 0.89 \\
\hline $\mathrm{ICH}$ & 17/107 (15.9\%) & $11 / 63(17.5 \%)$ & 0.95 \\
\hline $\mathrm{SDH}$ & 4/107 (3.7\%) & $2 / 63(3.2 \%)$ & 0.8 \\
\hline SAH & $3 / 107(2.8 \%)$ & $2 / 63(3.2 \%)$ & 0.75 \\
\hline Ischemic stroke & $44 / 107(41.1 \%)$ & $19 / 63(30.2 \%)$ & 0.2 \\
\hline Brain tumor & 8/107 (7.5\%) & 7/63 (11.1\%) & 0.6 \\
\hline Others & $31 / 107(29 \%)$ & $22 / 63(34.9 \%)$ & 0.5 \\
\hline
\end{tabular}

$\mathrm{NCCU}=$ neurocritical care unit; $\mathrm{SD}=$ standard deviation; $n=$ number; APACHE $=$ acute physiology and chronic health evaluation; $\mathrm{EDH}=$ extradural hemorrhage; $\mathrm{SDH}=$ subdural hemorrhage; $\mathrm{SAH}=$ subarachnoid hemorrhage; $\mathrm{ICH}=$ intracerebral hemorrhage; ${ }^{*}$ other neurological diagnoses included status epilepticus, encephalopathy, Guillain-Barré syndrome, and transverse myelitis.

TABle 2: Primary and secondary outcomes.

\begin{tabular}{lccc}
\hline & Group 1 & Group 2 & p value \\
\hline Primary outcomes & & & \\
ICU mortality $(n(\%))$ & $37(10.2 \%)$ & $11(5.3 \%)$ & \\
Hospital mortality $(n(\%))$ & $71(19.5 \%)$ & $19(9.1 \%)$ & \\
\hline Secondary outcomes & & & \\
ICU LOS (days; mean \pm SD) & $21.4 \pm 18.5$ & $15 \pm 12.5$ & 0.034 \\
Discharge GCS (mean \pm SD) & $11.5 \pm 2.6$ & $12.5 \pm 2.5$ & $<0.001$ \\
Tracheostomy $(n(\%))$ & $52(14.3 \%)$ & $28(13.5 \%)$ & 0.025 \\
ICP monitoring, $(n(\%))$ & $87(24 \%)$ & $112(53.8 \%)$ & 0.006 \\
Neurosurgical interventions $(n(\%))$ & $34(9.3 \%)$ & $41(19.7 \%)$ & $<0.001$ \\
\hline
\end{tabular}

$\mathrm{NCCU}=$ neurocritical care unit; ICU = intensive care unit; LOS= length of stay; GCS = Glasgow Coma Scale; ICP = intracranial pressure.

groups although lower in Group 2 (13\% in Group 1 and $8 \%$ in Group 2, $p=0.09)$. Sepsis and septic shock was the most common cause of hospital mortality in both groups $(56 \%$ in Group 1 and 55\% in Group 2). Since the majority of patients in both groups were trauma patients with high severity scores, $62 \%$ of Group 1 patients were mechanically ventilated as compared to $61 \%$ in Group $2(p=0.9) ; 61.5 \%$ of Group 1 patients required hemodynamic support $(>0.05 \mathrm{mcg} / \mathrm{kg} / \mathrm{min}$ noradrenaline) to maintain the BP targets of perfusion of the acutely injured brain and spinal cord, while $70.2 \%$ of Group 2 patients required hemodynamic support $(p=0.04)$; the rate of renal failure requiring hemodialysis at least once was not different between groups (31\% in Group 1 versus $27 \%$ in Group 2, $p=0.4$ ). Analysis of secondary outcomes revealed decreased ICU LOS in Group 2 compared to Group 1 $(p<0.001$; Table 2). Group 2 patients exhibited a higher ICU discharge GCS, underwent fewer tracheostomies but had more ICP monitoring and operative neurosurgical interventions compared to Group 1 patients (all $p<0.05$ ).
Two multivariate logistic regression models were fitted to evaluate independent predictors of ICU and/or hospital discharge among NCCU patients (evaluated for age, APACHE 4 score, gender, NCCU admission, presence of trauma, LOS, and operative intervention; Table 3). The models revealed that NCCU admission was not significantly correlated to ICU discharge $(\mathrm{OR}=1.5 ; 95 \% \mathrm{CI}$ : $0.71-3.3$; $p=0.285$ ) but was a significant predictor for hospital discharge with an OR of 2.3 (95\% CI: $1.3-4.1 ; p=0.005)$. Other significant predictors in both models were age and ICU LOS.

Both models were well fitted as $p$ values of the Hosmer-Lemeshow test were 0.28 and 0.67 in the multivariate logistic regression analysis, respectively. Both models were also well calibrated (evaluated the degree of correspondence between the estimated probabilities of mortality produced by a model and the actual mortality) as evident by the AUC of the logistic regression model for ICU discharge of 0.78 (95\% CI: 0.71-0.84) and that of the hospital discharge model of 0.74 (95\% CI: 0.68-0.8) (Figure 1). 
TABle 3: Predictors for ICU/hospital discharge.

\begin{tabular}{|c|c|c|c|c|}
\hline & Predictors & Odds ratio & $95 \% \mathrm{CI}$ & $p$ value \\
\hline \multirow{7}{*}{ ICU discharge } & NCCU admission & 1.5 & $0.7-3.3$ & 0.3 \\
\hline & Age (years) & 0.97 & $0.96-0.99$ & 0.02 \\
\hline & Gender (male/female) & 0.6 & $0.3-1.2$ & 0.13 \\
\hline & APACHE 4 score & 0.98 & $0.97-1$ & 0.051 \\
\hline & ICU LOS (days) & 0.97 & $0.96-0.98$ & 0.02 \\
\hline & Invasive procedures (\%) & 1.3 & $0.4-4$ & 0.6 \\
\hline & Presence of trauma & 0.9 & $0.4-1.8$ & 0.7 \\
\hline \multirow{7}{*}{ Hospital discharge } & NCCU admission & 2.3 & $1.3-4.1$ & 0.005 \\
\hline & Age (years) & 0.98 & $0.96-0.99$ & 0.001 \\
\hline & Gender (male/female) & 0.7 & $0.4-1.2$ & 0.2 \\
\hline & APACHE 4 score & 1 & $0.98-1.001$ & 0.064 \\
\hline & ICU LOS (days) & 0.98 & $0.97-0.99$ & 0.001 \\
\hline & Invasive procedures (\%) & 0.7 & $0.342-1.44$ & 0.335 \\
\hline & Presence of trauma & 1.3 & $0.8-2.2$ & 0.33 \\
\hline
\end{tabular}

$\mathrm{OR}=$ odds ratio; $\mathrm{CI}=$ confidence interval; $\mathrm{ICU}=$ intensive care unit; NCCU = neurocritical care unit; $\mathrm{APACHE}=$ acute physiology and chronic health evaluation; LOS = length of stay.

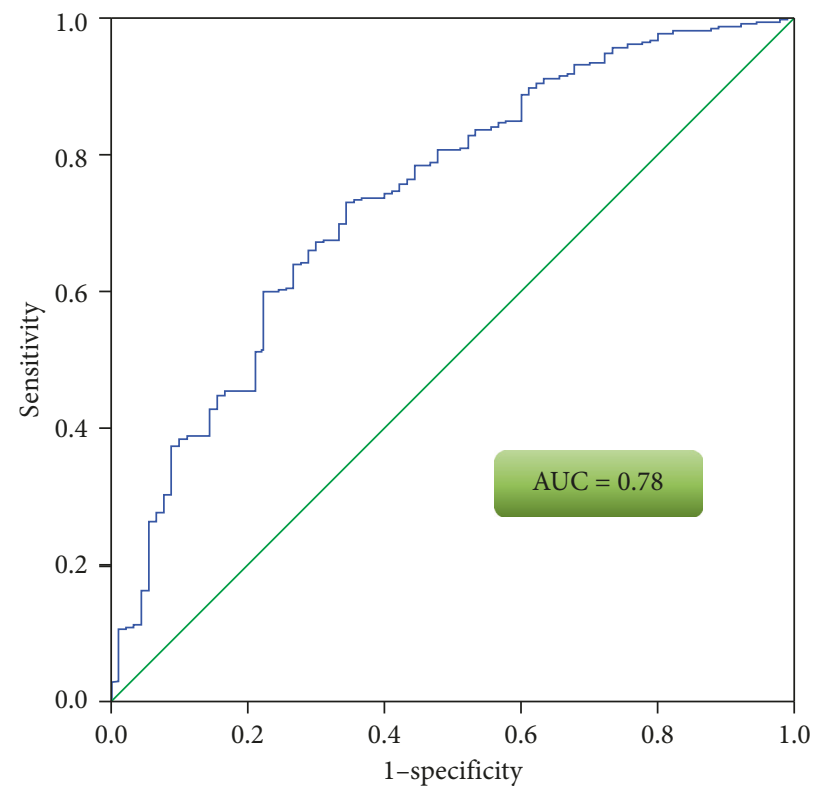

(a)

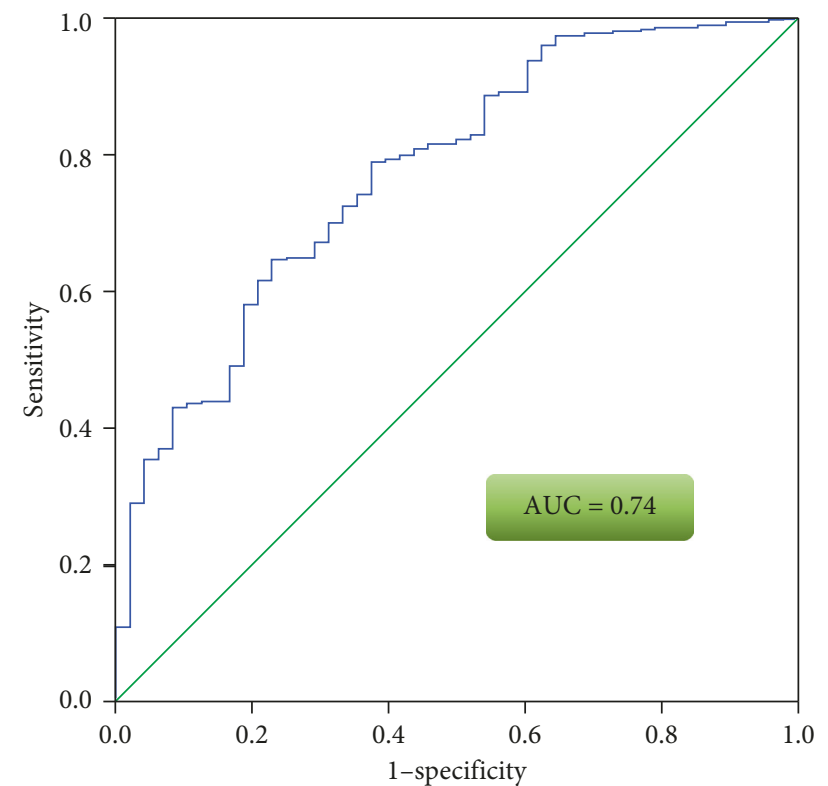

(b)

FIGURE 1: ROC curve of logistic regression models. (a) ICU discharge; (b) hospital discharge.

\section{Discussion}

In this retrospective before and after cohort study, we have shown that establishment of a dedicated NCCU was associated with an increase in meaningful clinical outcomes for neurologically injured patients.

The mortality rate $(5.3 \%)$ in the NCCU was significantly lower compared to the general ICU $(10.2 \%$; $p=0.034)$. This mirrors work by Jeong et al. [8]. Other larger studies have reported overall higher rates of NCCU mortality, such as $18 \%$ by Broessner et al. [23] ( $n=1000)$. Our hospital mortality rate was significantly reduced from $19.5 \%$ in general ICU to $9.1 \%$ $(p=0.001)$ in NCCU. This is also in accordance with findings of Varelas et al. [24], although other studies reported insignificant difference [25]. Adding to the significance of the reduced ICU and hospital mortality rates is the fact that, throughout the study period, there were no changes in our ICU's discharge policy or DNR policy. Furthermore, the general management of critically ill patients was consistent throughout the study period; however, the addition of new standards of management in the form of NCCU specific protocols to maintain better brain and spinal cord perfusion during acute injury guided by more ICP/CPP monitoring (evident by the significantly different rates of hemodynamic support requirement) may explain the difference in outcome objectively as adherence to guidelines was translated to more monitoring which guided us to maintain more perfusion of CNS by using more hemodynamic support in Group 2.

ICU LOS was significantly shorter for patients hospitalized in the NCCU compared to the general ICU $(p<0.001)$, although there were no changes in the setting or discharge policies of our institute nor was a step-down unit 
or new rehabilitation services established throughout the study period. This also duplicates what others have reported $[26,27]$. Notably, we did have a relatively high ICU LOS, which we believe can be largely attributed to the lack of a step-down unit in our institution. Notably, Kurtz et al. [10] reported a longer stay in NCCU, but this may reflect the binary model analysis used in his study, namely, patients were separated in two groups, those admitted less than or more than 10 days.

We found NCCU patients to have a better GCS at discharge. Importantly, many studies $[8,25]$ evaluate the Glasgow Outcome Scale (GOS) or modified Rankin Scale. Unfortunately, we lacked proper GOS data before the launching of NCCU. We accept this as a study limitation that was remedied by our new NCCU electronic medical records archive. Regardless, GCS at ICU discharge was significantly higher in the NCCU Group 2 compared to Group 1 $(p=0.025)$. Also, the rate of tracheostomies was significantly lower in the NCCU Group 2 compared to Group 1 $(p=0.006)$. While speculative, this could be partially attributed to the increased GCS, to better overall outcome, or to an evolving strategy towards less tracheostomies. In contrast, Kurtz et al. [10] reported that more NCCU patients $(35 \%)$ were receiving tracheostomy. However, we are unsure about their airway management strategy and intend to pursue this important question now that we have an established NCCU database. Despite the discrepant tracheostomy rates, our data are otherwise in agreement with Kurtz et al. [10] NCCU patients underwent closer neuromonitoring for secondary brain injuries clinically and through ICP insertions (parenchymal or ventricular) according to unit-specific protocols and guidelines for monitoring of different types of neurologic emergencies; hence, those protocols were applied after intensive educational and training activities for bedside nurses and physicians aiming for prevention and early detection and management of secondary injuries mainly intracranial hypertension $[8,10,22,23]$. The management included more neurosurgical interventions as craniotomies and decompressive craniectomies for refractory intracranial hypertension cases. However, the aforementioned finding does raise the question of whether more neurointerventions could be attributed to more intense neuromonitoring.

Multivariate models, when adjusted for age, gender, APACHE 4 score, LOS, trauma, and postoperative status, revealed that while NCCU admission was not an independent predictor of ICU discharge $(\mathrm{OR}=1.5$; $95 \% \mathrm{CI}$ : $0.71-3.3$; $p=0.285)$, it was a significant predictor of hospital discharge $(\mathrm{OR}=2.3$; 95\% CI of OR: $1.3-4.1 ; p=0.005)$. This is in accordance with other studies from other jurisdictions. Diringer and Edwards [13] reported that hospitalization outside of NCCU is associated with increased odds of in-hospital death (OR 3.4; 95\% CI: 1.65-7.6). Similarly, Suarez et al. [17] showed that the presence of NCC team is an independent predictor of decreased mortality (OR 0.7; 95\% CI: 0.5-1).

5.1. Limitations. This study has several limitations including its retrospective single-center design and the inherent weaknesses of any before and after analysis, as well as the aforementioned absence of GOS data or other neurological outcome measures such as modified Rankin Scale (mRS), as well as data of discharge disposition. With that said, we are excited to have shown such a positive impact associated with the establishment of a NCCU within a polyvalent ICU setting. Notably, our multivariate logistic regression analysis was tailored to evaluate general prognostic factors but did not include factors specific for particular neurological conditions such as ICH volume/score, SAH grade, and ischemic stroke type/size.

Finally, the age and gender distribution-although similar in both groups-revealed a preponderance of males with a mean age of about 40 years, that is consistent with previous studies on road traffic accident victims in Saudi Arabia [28] who constitute the majority of our patients, a finding that is although typical of the milieu in Saudi Arabia may affect the generalizability of our findings. Further larger prospective multicenter studies are clearly required to confirm and establish the generalizability of our findings.

\section{Conclusion}

Creation of a dedicated NCCU was associated with a significant reduction in ICU and hospital mortality rates, as well as ICU LOS. Admission to NCCU was an independent predictor of discharge from the hospital. NCCU-discharged brain-injured patients exhibited higher GCS and required more frequently invasive neuromonitoring and other interventional procedures with the notable exemption of performed tracheostomies.

\section{Conflicts of Interest}

The authors declare no conflicts of interest.

\section{Authors' Contributions}

Ibrahim Soliman helped to form the conceptual framework for this article and to conduct the literature search, participated in interpretation of data, and shared responsibility for the tables. Waleed Tharwat Aletreby helped to form the conceptual framework for this article, to conduct the literature search, and performed the statistical analysis. Fahad Faqihi, Nasir Nasim Mahmood, Omar E. Ramadan, and Ahmad Fouad Mady participated in the literature search, data collection, and interpretation. Babar Kahlon, Abdulrahman Alharthy, and Peter Brindley helped in quality control and statistical analysis. Dimitrios Karakitsos conducted critical revision of the manuscript and supervised the study. All authors contributed to the drafting, revising, and approval of the final manuscript.

\section{Acknowledgments}

The authors would like to acknowledge the efforts of all staff at Critical Care Department, King Saud Medical City, Riyadh, Kingdom of Saudi Arabia. 


\section{References}

[1] M. S. Sekhon, P. Gooderham, B. Toyota, and N. Kherzi, "Implementation of neurocritical care is associated with improved outcomes in traumatic brain injury," Canadian Journal of Neurological Sciences, vol. 44, no. 4, pp. 350-357, 2017.

[2] L. Knopf, I. Staff, J. Gomes, and L. McCullough, "Impact of a neurointensivist on outcomes in critically ill stroke patients," Neurocritical Care, vol. 16, no. 1, pp. 63-71, 2012.

[3] I. C. Kiphuth, P. D. Schellinger, M. Köhrmann et al., "Predictors for good functional outcome after neurocritical care," Critical Care, vol. 14, no. 4, p. R136, 2010.

[4] I. Tweedie, "Neuro-critical care versus general critical care for neurological injury: beneficial evidence," Journal of Neuroanaesthesiology and Critical Care, vol. 3, no. 4, pp. 62-65, 2016.

[5] Y. Kuroda, "Neurocritical care update," Journal of Intensive Care, vol. 4, no. 1, p. 36, 2016.

[6] A. H. Kramer and D. A. Zygun, "Do neurocritical care units save lives? measuring the impact of specialized ICUs," Neurocritical Care, vol. 14, no. 3, pp. 329-333, 2011.

[7] D. A. Zygun, J. B. Kortbeek, G. H. Fick, K. B. Laupland, and C. J. Doig, "Nonneurologic organ dysfunction in severe traumatic brain injury," Critical Care Medicine, vol. 33, no. 3, pp. 654-660, 2005.

[8] J.-H. Jeong, J. S. Bang, W. J. Jeong et al., "A Dedicated neurological intensive care unit offers improved outcomes for patients with brain and spine injuries," Journal of Intensive Care Medicine, article 088506661770667, 2017.

[9] J.-H. Jeong, M. Yeo, J.-H. Hong, K. S. Yum, J. Y. Chang, and M.-K. Han, "Clinical outcomes of the first neurocritical care unit in Korea," Stroke, vol. 47, article ATP437, 2016.

[10] P. Kurtz, V. Fitts, Z. Sumer et al., "How does care differ for neurological patients admitted to a neurocritical care unit versus a general ICU?," Neurocritical Care, vol. 15, no. 3, pp. 477-480, 2011.

[11] P. N. Varelas, D. Eastwood, H. J. Yun et al., "Impact of a neurointensivist on outcomes in patients with head trauma treated in a neurosciences intensive care unit," Journal of Neurosurgery, vol. 104, no. 5, pp. 713-719, 2006.

[12] K. Kyeremanteng, A. Hendin, K. Bhardwaj et al., "Neuroscience intermediate-level care units staffed by intensivists: clinical outcomes and cost analysis," Journal of Intensive Care Medicine, article 088506661770665, 2017.

[13] M. N. Diringer and D. F. Edwards, "Admission to a neurologic/neurosurgical intensive care unit is associated with reduced mortality rate after intracerebral hemorrhage," Critical Care Medicine, vol. 29, no. 3, pp. 635-640, 2001.

[14] E. M. Bershad, E. S. Feen, O. H. Hernandez, M. F. Suri, and J. I. Suarez, "Impact of a specialized neurointensive care team on outcomes of critically ill acute ischemic stroke patients," Neurocritical Care, vol. 9, no. 3, pp. 287-292, 2008.

[15] S. Egawa, T. Hifumi, K. Kawakita et al., "Impact of neurointensivist-managed intensive care unit implementation on patient outcomes after aneurysmal subarachnoid hemorrhage," Journal of Critical Care, vol. 32, pp. 52-55, 2016.

[16] A. Oommen, "Neuro critical care-how it makes a difference in neurology," Journal of Neurology and Stroke, vol. 2, no. 1, article 00045, 2015.

[17] J. I. Suarez, O. O. Zaidat, M. F. Suri et al., "Length of stay and mortality in neurocritically ill patients: impact of a specialized neurocritical care team," Critical Care Medicine, vol. 32, pp. 2311-2317, 2004.
[18] P. N. Varelas, T. Abdelhak, J. Wellwood, D. Benczarski, S. B. Elias, and M. Rosenblum, "The appointment of neurointensivists is financially beneficial to the employer," Neurocritical Care, vol. 13, no. 2, pp. 228-232, 2010.

[19] N. Carney, A. M. Totten, C. O'Reilly et al., "Guidelines for the management of severe traumatic brain injury, fourth edition," Neurosurgery, vol. 80, no. 1, pp. 6-15, 2017.

[20] W. J. Powers, A. A. Rabinstein, T. Ackerson et al., "2018 Guidelines for the early management of patients with acute ischemic stroke: a guideline for healthcare professionals from the American Heart Association/American Stroke Association," Stroke, vol. 49, no. 3, pp. e46-e110, 2018.

[21] G. D. Ruxton, "The unequal variance $t$-test is an underused alternative to student's $t$-test and the Mann-Whitney $U$ test," BMC Ecology, vol. 17, no. 4, pp. 688-690, 2006.

[22] C. M. Rodrigues, E. M. C. Pires, J. P. O. Feliciano, J. M. Vieira Jr., and L. U. Taniguchi, "Admission factors associated with intensive care readmission in critically ill oncohematological patients: a retrospective cohort study," Revista Brasileira de Terapia Intensiva, vol. 28, no. 1, pp. 33-39, 2016.

[23] G. Broessner, R. Helbok, P. Lackner et al., "Survival and longterm functional outcome in 1,155 consecutive neurocritical care patients," Critical Care Medicine, vol. 35, no. 9, pp. 2025-2030, 2007.

[24] P. N. Varelas, M. M. Conti, M. V. Spanaki et al., "The impact of a neurointensivist-led team on a semiclosed neurosciences intensive care unit," Critical Care Medicine, vol. 32, no. 11, pp. 2191-2198, 2004.

[25] J.-A. Ryu, J. H. Yang, C. R. Chung, G. Y. Suh, and S.-C. Hong, "Impact of neurointensivist co-management on the clinical outcomes of patients admitted to a neurosurgical intensive care unit," Journal of Korean Medical Science, vol. 32, no. 6, pp. 1024-1030, 2017.

[26] Y. Kim, S.-B. Kwon, H.-J. Park et al., "Predictors of functional outcome of patients in neurological intensive care unit," Neurobiology of Aging, vol. 17, no. 3, pp. 219-225, 2012.

[27] S. A. Josephson, V. C. Douglas, M. T. Lawton, J. D. English, W. S. Smith, and N. U. Ko, "Improvement in intensive care unit outcomes in patients with subarachnoid hemorrhage after initiation of neurointensivist co-management," Journal of Neurosurgery, vol. 112, no. 3, pp. 626-630, 2010.

[28] F. A. Mansuri, A. H. Al-Zalabani, M. M. Zalat, and R. I. Qabshawi, "Road safety and road traffic accidents in Saudi Arabia: A systematic review of existing evidence," Saudi Medical Journal, vol. 36, no. 4, pp. 418-424, 2015. 


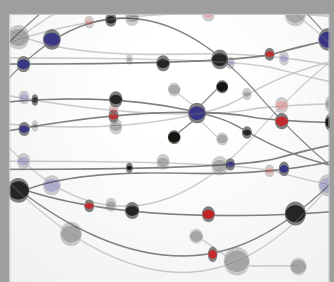

The Scientific World Journal
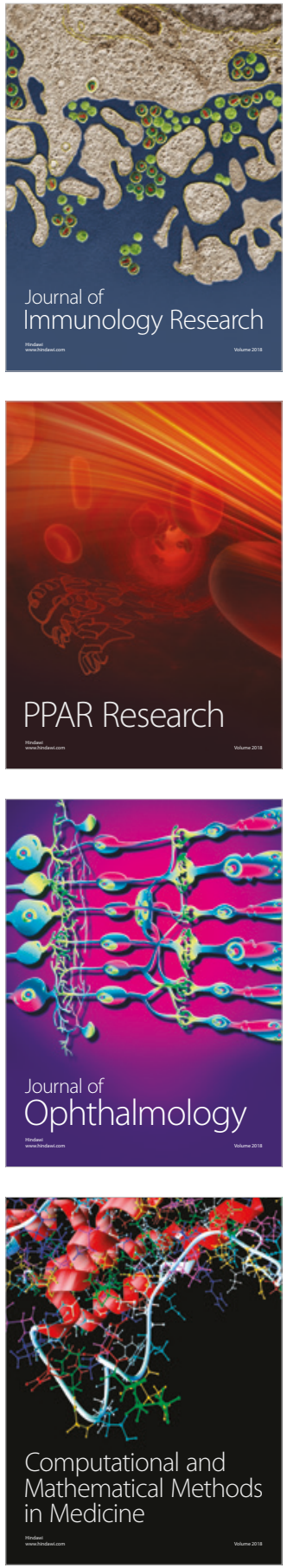

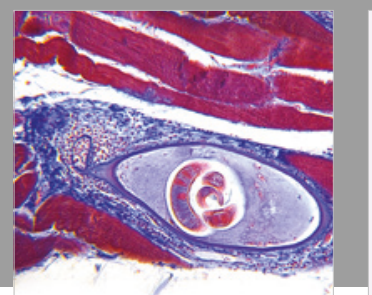

Gastroenterology Research and Practice

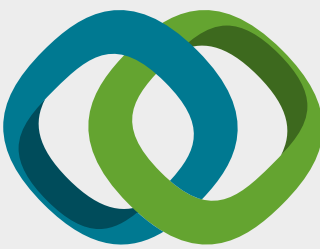

\section{Hindawi}

Submit your manuscripts at

www.hindawi.com
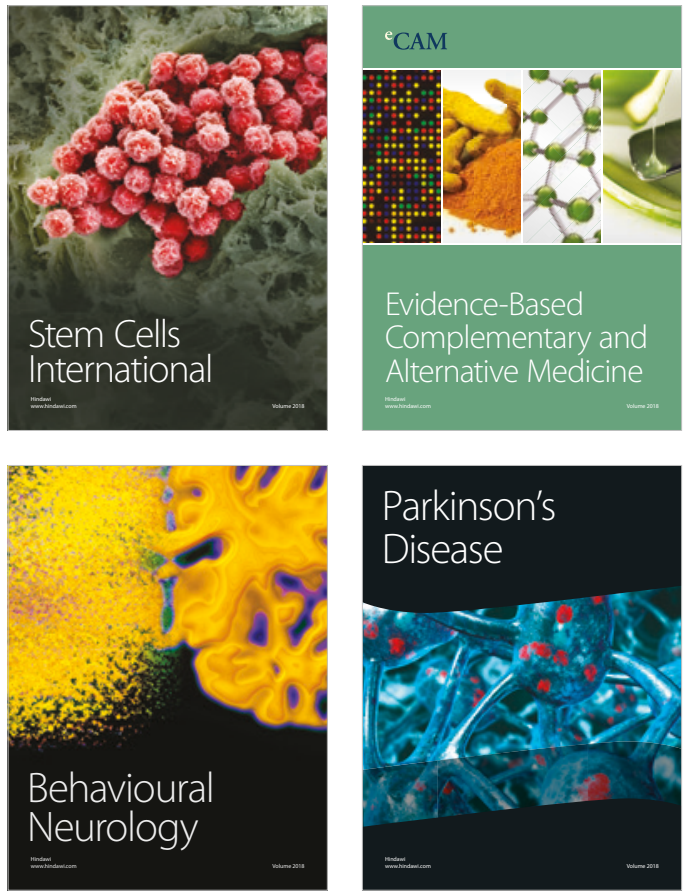

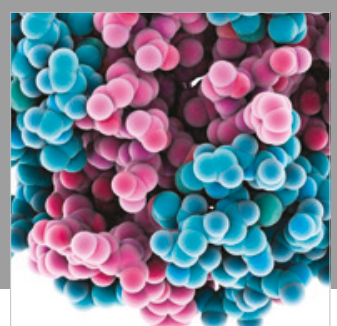

ournal of

Diabetes Research

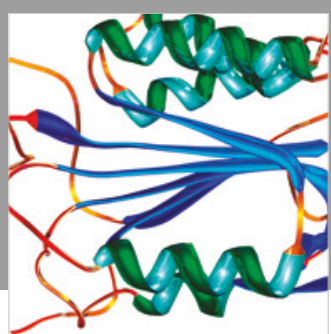

Disease Markers
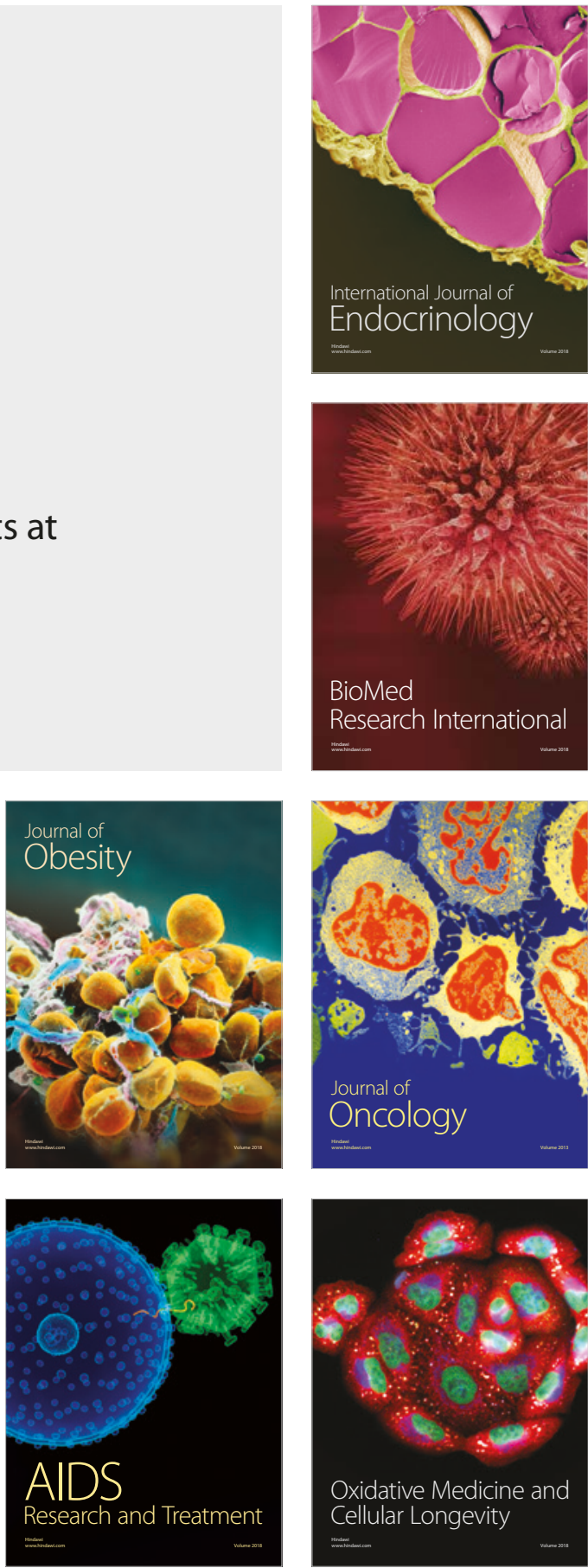